\title{
Automated Evaluation of the Kinetics of Damage Accumulation on the Surface of a Fatigue Sensor Based on the Analysis of...
}

Article in Optoelectronics Instrumentation and Data Processing · July 2014

DOI: $10.3103 /$ S8756699014040050

CITATIONS

2

4 authors:

Ihor Konovalenko

Ternopil State Ivan Pul'uj Technical University

42 PUBLICATIONS 144 CITATIONS

SEE PROFILE

\section{Pavlo Maruschak}

Ternopil Ivan Pul'uj National Technical University 178 PUBLICATIONS 511 CITATIONS

SEE PROFILE
READS

54
Mikhail Karuskevich

National Aviation University

40 PUBLICATIONS 86 CITATIONS

SEE PROFILE

\section{Sergey Ignatovich}

National Aviation University

55 PUBLICATIONS 83 CITATIONS

SEE PROFILE

Some of the authors of this publication are also working on these related projects:

Project Surface relief as an indicator of accumulated fatigue damage View project 


\title{
Automated Evaluation of the Kinetics of Damage Accumulation on the Surface of a Fatigue Sensor Based on the Analysis of Strain Relief Parameters
}

\author{
I. B. Konovalenko ${ }^{a}$, M. V. Karuskevich ${ }^{b}$, P. O. Marushchak ${ }^{a}$, \\ and S. R. Ignatovitch ${ }^{b}$ \\ ${ }^{a}$ Ternopil Ivan Pul'uj National Technical University, \\ ul. Russkaya 56, Ternopil, 46001 Ukraine \\ ${ }^{b}$ National Aviation University, \\ pr. Kosmonavta Komarova 1, Kiev, 03058 Ukraine \\ E-mail: Maruschak.tu.edu@gmail.com
}

Received March 14, 2013

\begin{abstract}
Digital identification and evaluation of the kinetics of damage accumulation on the surface of a fatigue sensor are performed using digital image processing. Damage accumulation is evaluated from the results of diagnosis of individual stages of cyclic deformation. It is found that the different stages of damage correspond to particular image parameters. The main features in the development of shear processes were identified by consistent processing of surface damage data. Theoretical background and experimental results are presented.
\end{abstract}

Keywords: cyclic loading, strain relief, image analysis, diagnosis.

DOI: $10.3103 / \mathrm{S} 8756699014040050$

\section{INTRODUCTION}

It is known that the kinetics of fatigue damage accumulation and failure mechanisms of materials are determined by the internal structure of material and its degradation during operation time [1]. Therefore, studies of damage accumulation and plastic strain localization at different scale levels make it possible to evaluate the kinetics of the process and predict the limit state of materials [2]. These processes have been studied in a number of papers [3-5], which proved the physical background and technical implementation of structure-sensitive fatigue sensors for aircraft. These sensors can be effectively used to assess the actual state of aircraft structures by high-precision non-destructive methods.

The methodology of large-scale and structural levels is presented in [6-8], together with the concept of elementary carriers of deformation which considers material deformation as a hierarchical system prone to structural-mechanical self-organization. This approach underlies numerical algorithms for estimating the state of systems and analysis of damage detected in the surface layer [9, 10]; it also provides a background for evaluating the actual technical state of an object between scheduled inspections.

In $[11,12]$, two-dimensional, three-dimensional, and fractal parameters of strain relief are established and deformation structures and their corresponding levels of accumulated fatigue damage are quantitatively described. Structure-sensitive witness samples (sensors) of fatigue damage have been designed [4], whose use is based on a quantitative evaluation of the accumulated fatigue damage from strain surface relief parameters. The sensitivity of witness samples can be optimized in accordance with the conditions of operational cyclic loading of structures [4]. This necessitates the design of effective algorithms for the identification, analysis, and synthesis of surface damage data taking into account the orientation of defects $[13,14]$.

The purpose of this study was to design an algorithm for the automated evaluation of the fatigue damage of a foil sensor as a nonlinear hierarchically organized structural-mechanical system. 
(a)

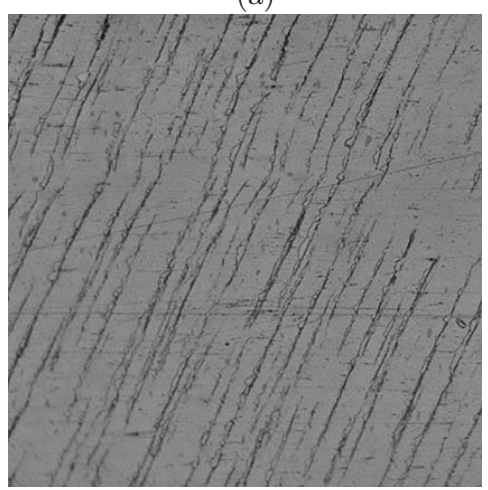

(d)

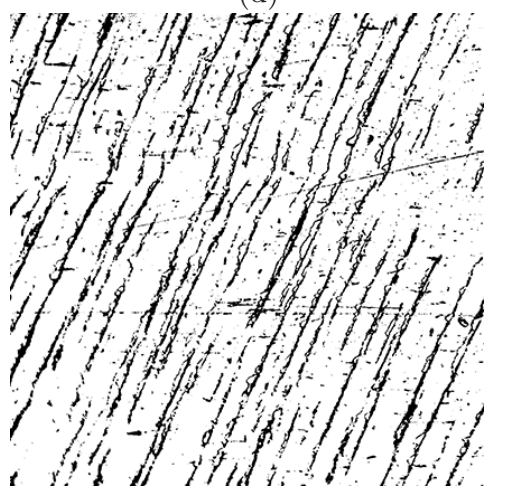

(g)

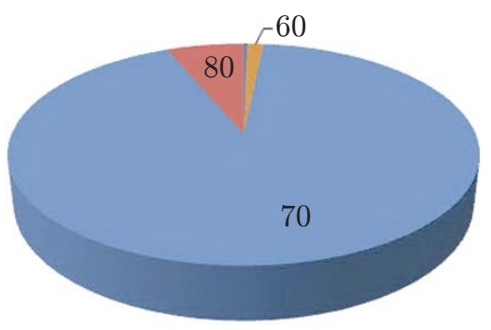

(b)

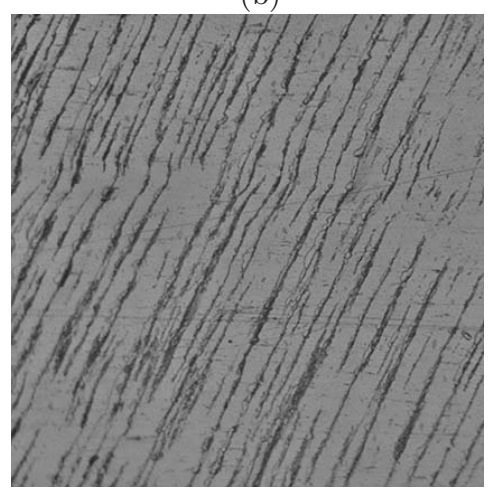

(e)

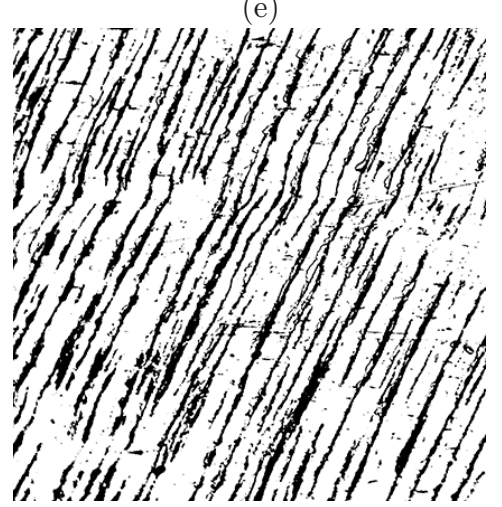

(h)

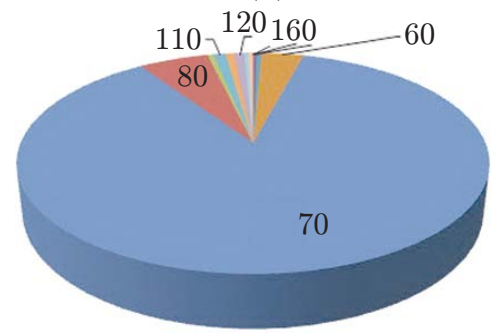

(c)

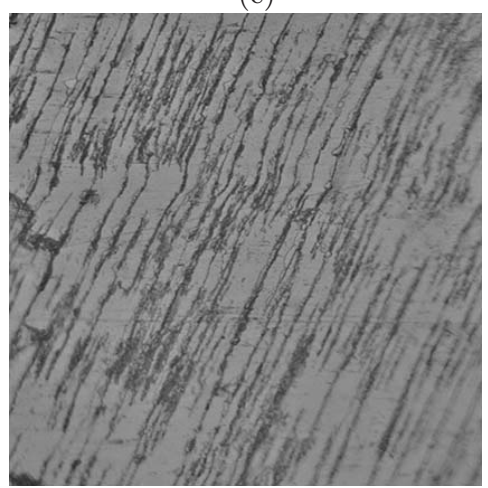

(f)

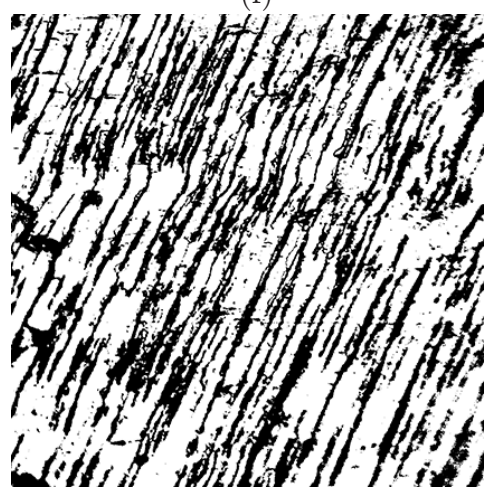

(i)

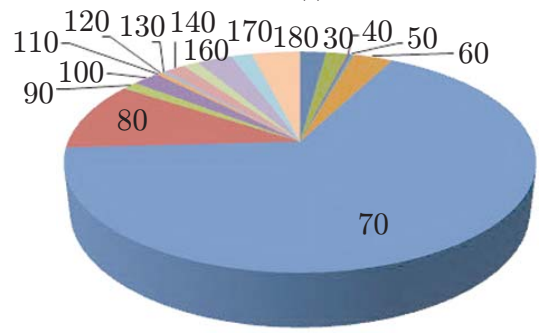

Fig. 1. Surface of a polycrystalline aluminum fatigue sensor and data of its analysis after $10^{4}, 4 \cdot 10^{4}$, and $2 \cdot 10^{5}$ loading cycles (columns, respectively): original multigradation $(\mathrm{a}-\mathrm{c})$ and the final thresholded $(\mathrm{d}-\mathrm{f})$ images and distribution diagrams of the angles of orientation of relief structures $(\mathrm{g}-\mathrm{i})$.

\section{PHYSICOMECHANICAL BACKGROUND FOR DAMAGE DIAGNOSIS}

Any damaged surface can be regarded as a set of structural defects located within the investigated region. Here defects are manifestations of microshear and rotation processes, damage of grain boundaries, etc. Increased cyclic operation usually leads to the occurrence of slip lines and their development with the formation of localized shear structures, which is one of the ways of stress relaxation under cyclic loading. A foil sensor was fixed on a specimen of D16AT alloy, which was tested at a maximum stress $\sigma_{\max }=180.0 \mathrm{MPa}$ at a frequency of $11.0 \mathrm{~Hz}$ and cycle asymmetry [11]. The fatigue sensor allows monitoring of the kinetics of accumulation and localization of fatigue damage in the surface layer of the material using methods of physical mesomechanics. An elementary act of plastic flow during fatigue damage accumulation in material is the shear + rotation of structural elements [6]. This leads to the formation of dissipative mesostructures and the accumulation of fatigue defects in fatigue sensors.

\section{ALGORITHM FOR CALCULATING STRAIN RELIEF PARAMETERS}

Surface relief parameters of the polycrystalline aluminum fatigue sensor were determined by analyzing the original image $I_{0}(x, y)($ Fig. $1 \mathrm{a}-\mathrm{c})$.

OPTOELECTRONICS, INSTRUMENTATION AND DATA PROCESSING Vol. 50 No. 42014 


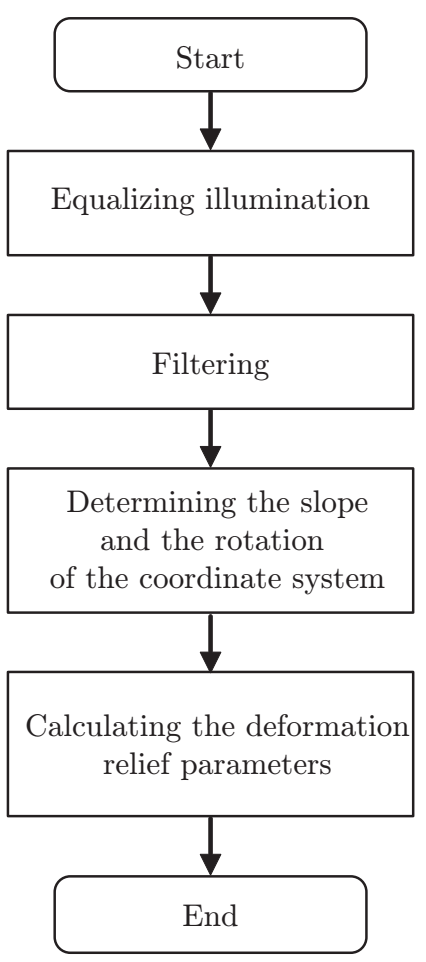

Fig. 2. Block diagram of the algorithm for determining strain relief parameters.

The algorithm consists of two steps (see Fig. 2). The first, preparatory, step comprises the operations of equalizing illumination, filtering, thresholding, and determining the slope (see Fig. 1d-f). In the second step, quantitative integral characteristics providing an insight into the general state of the sensor were calculated by analyzing the image obtained [2]. It should be noted that special attention was paid to monitoring of the disorientation of the shear bands, which is one of the fundamental manifestations of the kinetic regularities of damage accumulation [6-8]. Local microshears in some areas of the bands cause a change in their integral orientation. There is a transition from the uniformly oriented structure of shears to a partially disoriented structure with a gradual increase in the amount of microdistortions of the strain relief geometry.

This phenomenon is confirmed by a diagram of the orientation of the relief structures (see Fig. 1g-i). It is seen that the deformation structures are first unidirectional, and then local relaxation processes at the mesolevel give rise to self-organization mechanisms. The angles of propagation of relief structures were analyzed using an identification algorithm [15].

\section{Image Pre-Preprocessing}

The influence of the shaded areas of the original image on the analysis results was eliminated to equalize the image illumination. This operation was performed by convolution of the image using a low-pass filter with the subsequent elimination of the low-frequency component.

A Gaussian filter with a sufficiently large kernel size was used for filtering, resulting in an approximate image of illumination $l^{\prime}(x, y)$ (where $x$ is the column index, $x \in(1, \ldots, m) ; y$ is the image row index $I_{0}$, $y \in(1, \ldots, n))$.

After that we have an image with equalized illumination

$$
I_{L}(x, y)=K_{L} \frac{I_{0}(x, y)}{l^{\prime}(x, y)},
$$

where $K_{L}=\max \left(l^{\prime}(x, y)\right)$ is the illumination equalization coefficient.

A median filter was used to eliminate minor noise in the final image, caused by the operation features of the photomatrix. The result of processing was the image $I_{f}$.

OPTOELECTRONICS, INSTRUMENTATION AND DATA PROCESSING Vol. $50 \quad$ No. 42014 
After pre-preprocessing and improving the quality of the original image $I_{0}$, we segmented the image by thresholding. In the image, informative pixels (points of interest) corresponding to strain relief regions were identified.

For this, in the image brightness diagram, the boundary value $I_{B_{\lim }}$ separating the background pixels from the informative pixels was determined. The result is the binary image $I$ (see Fig. $1 \mathrm{~d}-\mathrm{f}$ ) in which black pixels (informative) correspond to the strain relief elements, and white pixels to the background elements.

Strain relief on the surface of the polycrystalline aluminum fatigue sensor is characterized by multiple linear fragments (see Fig. 1). A prerequisite for obtaining integral quantitative indicators of such images [2] is a coordinate system one of whose axes coincides with the direction of propagation of linear fragments of the strain relief.

\section{Rotation of the Coordinate Axes}

The direction of propagation of linear fragments of the strain relief was determined using a Hough transform, in which the image is compared with the parameter space of straight lines [16]. Let the family of straight lines in the plane be described by the parameter equation

$$
h(\theta, \rho, x, y)=x \cos \theta+y \sin \theta-\rho,
$$

where $(x, y)$ is the parameter space of the image $I ;(\theta, \rho)$ is the parameter space of the family of straight lines presented in the image ( $\theta$ and $\rho$ are the components of the normal equation of the straight line).

In the Hough transform, the matrix of accumulators $S$ in the space of parameters $(\theta, \rho)$ was calculated with discreteness $\Delta \theta, \Delta \rho$ (in the image analysis (see Fig. 1), it was assumed that $\Delta \theta=1^{\circ}$ and $\Delta \rho=4$ pixels). Each cell of the phase space corresponds to an accumulator with a certain number of informative points in the image. It was assumed that the assignment of a point $i(x, y)$ of an image $I$ to a straight line $h$ is given by a cell of the parameter space $S(\theta, \rho)$ :

$$
r(x, y, \theta, \rho)= \begin{cases}1, & \text { if } d(i(x, y), h(\theta, \rho, x, y)) \leq d_{\lim } \\ 0 & \text { otherwise }\end{cases}
$$

where $d(i(x, y), h(\theta, \rho, x, y))$ is the distance from the point $i(x, y)$ to the straight line $h(\theta, \rho, x, y) ; d_{\lim }$ is the boundary value.

Thus, the Hough transform function has the form

$$
H(S(\theta, \rho))=\sum r(x, y, \theta, \rho)
$$

Straight lines with the largest number of informative points were found in the image by quantitative analysis of the accumulators in the parameter space. The zones in which the function $H(S(\theta, \rho))$ reaches maximum values correspond to more pronounced straight lines. The predominant direction of propagation of informative points (strain relief elements) are obtained from the value of the parameter $\theta=\theta_{\text {base }}$. The form of the function $H(S(\theta, \rho))$ for the image in Fig. 1e is shown in Figs. 3 and 4.

Next, we considered the image $I$ in the coordinate system $\left(x^{\varphi}, y^{\varphi}\right)$ after rotation about the origin of the coordinate axes $(x, y)$ by an angle $\varphi=\theta_{\text {base }}-\pi / 2$ (see Fig. 4 ). For the images shown in Fig. 1e, the angle $\theta_{\text {base }}=0.47 \mathrm{rad}=27^{\circ}$.

Note that the predominant slope of the relief structures can be calculated only in the case of an elongated shape and the trend toward defect orientation in a certain direction. Otherwise, the parameter plane of the Hough transform will not contain pronounced zones that corresponds to a certain angle. Then, the step of the algorithm with the rotation of the coordinate system can be skipped by going directly to the calculation of the quantitative parameters in the initial coordinate system of the image without pronounced differences for both coordinate axes.

OPTOELECTRONICS, INSTRUMENTATION AND DATA PROCESSING Vol. 50 No. 42014 


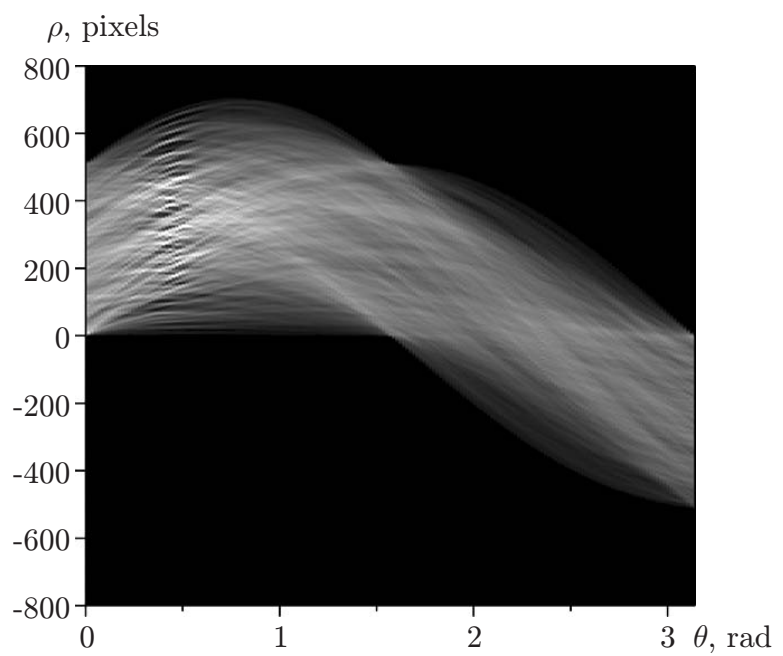

Fig. 3. Form of the function $H(S(\theta, \rho))$ for the analyzed strain relief.

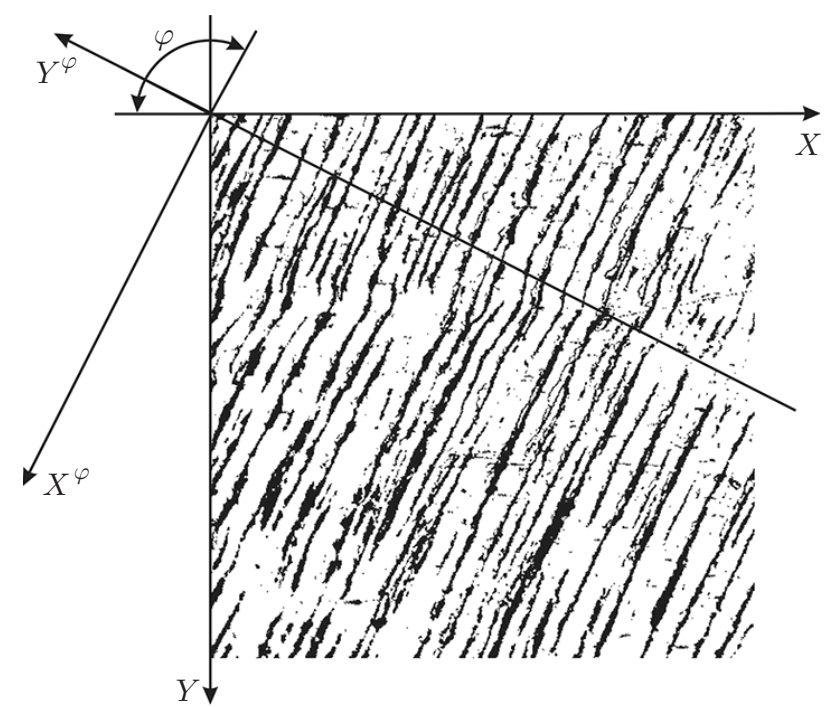

Fig. 4. Rotation of the coordinate system of the image in the direction of development of the strain relief elements.

\section{QUANTITATIVE ESTIMATION OF THE RELIEF PARAMETERS}

For a quantitative estimation of the strain relief of the surface of the fatigue sensor, we used a number of parameters calculated by analyzing the processed multigradation image $I_{f}$ and the binary image $I$.

For each image pixel $I_{f}$, the absolute values of the horizontal and vertical gradients were determined:

$$
\nabla I_{f}^{h}\left(x^{\varphi}, y^{\varphi}\right)=\left|\frac{\partial I_{f}\left(x^{\varphi}, y^{\varphi}\right)}{\partial x^{\varphi}}\right| ; \quad \nabla I_{f}^{v}\left(x^{\varphi}, y^{\varphi}\right)=\left|\frac{\partial I_{f}\left(x^{\varphi}, y^{\varphi}\right)}{\partial y^{\varphi}}\right| .
$$

A generalized estimate of the surface under study was obtained using the average value of the horizontal and vertical gradients of the image:

$$
G_{h}=\overline{\nabla I_{f}^{h}}=\frac{1}{S_{I}} \iint \nabla I_{f}^{h}\left(x^{\varphi}, y^{\varphi}\right) d x^{\varphi} d y^{\varphi} ; \quad G_{v}=\overline{\nabla I_{f}^{v}}=\frac{1}{S_{I}} \iint \nabla I_{f}^{v}\left(x^{\varphi}, y^{\varphi}\right) d x^{\varphi} d y^{\varphi},
$$

where $S_{I}=m n$ is the image area. 
(a)

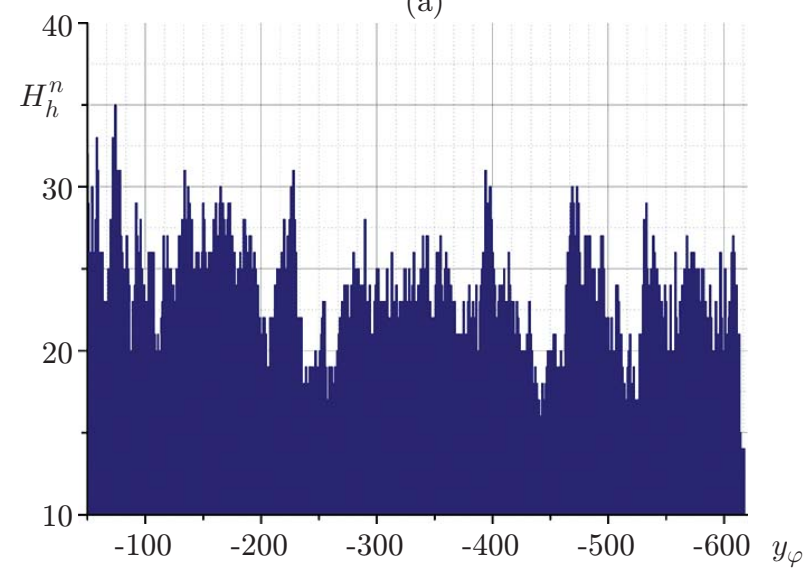

(b)

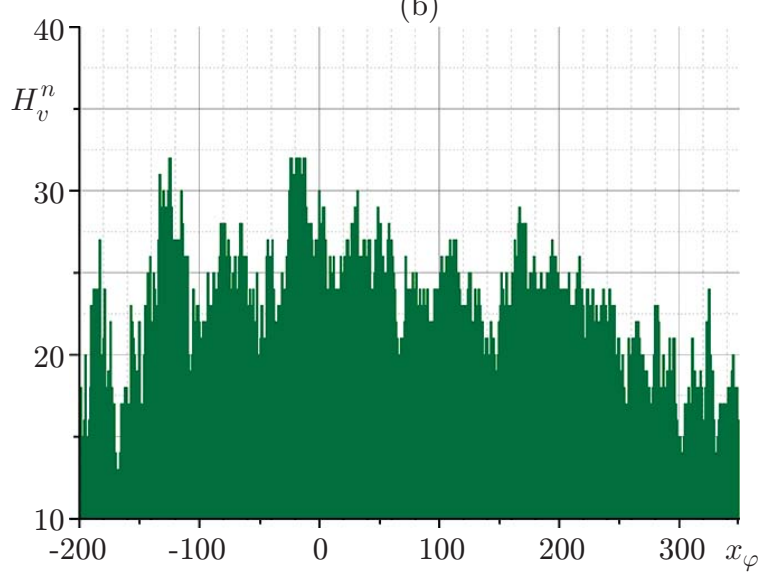

Fig. 5. Histograms of the image of the surface of the polycrystalline aluminum fatigue sensor: normalized horizontal $H_{h}^{n}(a)$ and vertical $H_{h}^{n}(b)$.

The gradient allows a quantitative estimation of the degree of heterogeneity of the investigated surface along the coordinate axes. A low gradient indicates a slight change in the intensity along the axis of the image considered. In practice, this implies a more uniform pattern of ordered structures in a certain direction [13], and indicates a coordinate axis that corresponds to the predominant direction of damage. An integral parameter that can be used to analyze the state of the sensor is the relative area of damage

$$
S_{d}=\frac{S}{m n} \cdot 100 \%,
$$

where $S$ is the number of pixels of the ordered structures of the image $I$.

The distribution of the elements of ordered structures along the axes $\left(X^{\varphi}, Y^{\varphi}\right)$ of the image is described by a vertical $H_{v}$ and a horizontal $H_{h}$ histograms [2]:

$$
H_{h}\left(y^{\varphi}\right)=\sum_{x^{\varphi}=1}^{m^{\varphi}} I\left(x^{\varphi}, y^{\varphi}\right) ; \quad H_{v}\left(x^{\varphi}\right)=\sum_{y^{\varphi}=1}^{n^{\varphi}} I\left(x^{\varphi}, y^{\varphi}\right)
$$

where $m^{\varphi}$ and $n^{\varphi}$ are the image dimensions in the coordinate system $\left(x^{\varphi}, y^{\varphi}\right)$ along the axes $X^{\varphi}$ and $Y^{\varphi}$, respectively.

Given that after the rotation of the coordinate system, the sections of the image for different values of $x^{\varphi}$ (or $y^{\varphi}$ ) will be different, we normalized the histograms to perform a comparative analysis:

$$
H_{h}^{n}\left(y^{\varphi}\right)=\frac{H_{h}\left(y^{\varphi}\right)}{d_{I}\left(y^{\varphi}\right)} \cdot 100 \% ; \quad H_{v}^{n}\left(x^{\varphi}\right)=\frac{H_{v}\left(x^{\varphi}\right)}{d_{I}\left(x^{\varphi}\right)} \cdot 100 \%,
$$

where $d_{I}\left(y^{\varphi}\right)$ and $d_{I}\left(x^{\varphi}\right)$ are the image dimensions along the axes $Y^{\varphi}$ and $X^{\varphi}$.

Each element of the histogram contains a number of points that correspond to the ordered structures of the relief in the horizontal and vertical directions of the analyzed image. The functions of the histograms contain the basic array of information on the distribution of the ordered structures along the coordinate axes of the image. The normalized histograms for the investigated image of the surface of the polycrystalline aluminum fatigue sensor (see Fig. 1e) are shown in Fig. 5. Note that the horizontal histogram is characterized by pronounced peaks corresponding to blocks of unidirected relief elements. At the same time, the vertical histogram has a more uniform shape, which indicates the absence of pronounced linear elements in this direction. A quantitative estimate of the form of histograms (8) was obtained by spectral analysis of their functions. Using the fast Fourier transform, the histogram functions can be written as the series

$$
H_{h}^{n}\left(y^{\varphi}\right) \approx \sum_{k=0}^{K_{h}} A_{h k} \cos \left(2 \pi \frac{k}{n} y^{\varphi}-\theta_{h}\right) ; \quad H_{v}^{n}\left(x^{\varphi}\right) \approx \sum_{k=0}^{K_{v}} A_{v k} \cos \left(2 \pi \frac{k}{m} x^{\varphi}-\theta_{v}\right) .
$$


Table 1. Parameter values of the surface of the fatigue sensor for different operation times

\begin{tabular}{c|c|c|c|c}
\hline \multirow{2}{*}{$\begin{array}{c}\text { Number } \\
\text { of operation cycles } \\
N\end{array}$} & \multicolumn{2}{|c|}{ Gradient } & \multicolumn{2}{c}{ Average amplitude of the spectrum } \\
\cline { 2 - 5 } & $\begin{array}{c}\text { horizontal } \\
G_{h}\end{array}$ & $\begin{array}{c}\text { vertical } \\
G_{v}\end{array}$ & $\begin{array}{c}\text { horizontal } \\
A_{a h}\end{array}$ & $\begin{array}{c}\text { vertical } \\
A_{a v}\end{array}$ \\
\hline 10000 & 12.0 & 17.6 & 0.5 & 0.6 \\
\hline 40000 & 16.1 & 28.8 & 0.7 & 0.9 \\
\hline 200000 & 15.8 & 27.9 & 1.3 & 2.0 \\
\hline
\end{tabular}

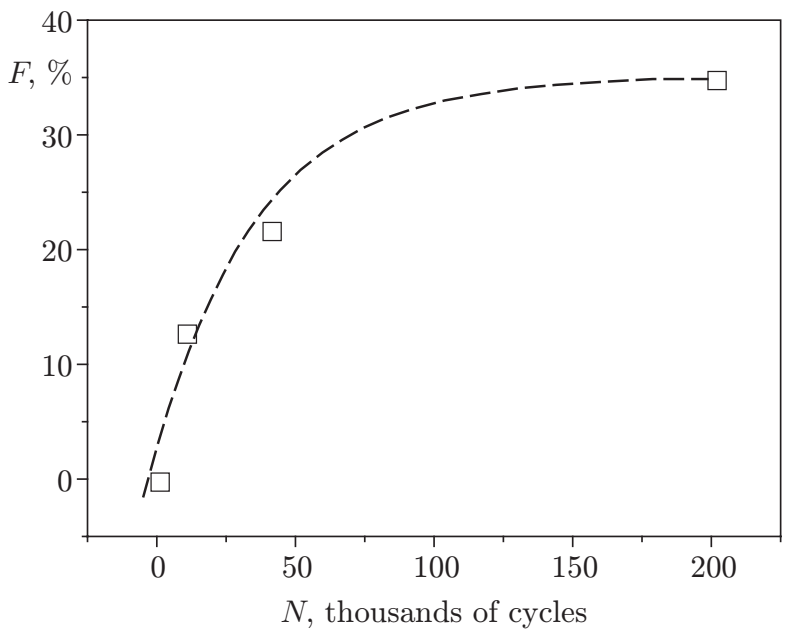

Fig. 6. Change in the damage area $F$ during operation $N$.

The numbers of harmonics $K_{h}$ and $K_{v}$ were chosen so as to represent the histogram function as a sum of harmonics not less than the boundary value of $\varepsilon$ :

$$
\left|H_{h}^{n}\left(y^{\varphi}\right)-\sum_{k=0}^{K_{h}} A_{h k} \cos \left(2 \pi \frac{k}{n} y^{\varphi}-\theta_{h}\right)\right| \leq \varepsilon ; \quad\left|H_{v}^{n}\left(x^{\varphi}\right)-\sum_{k=0}^{K_{v}} A_{v k} \cos \left(2 \pi \frac{k}{m} x^{\varphi}-\theta_{v}\right)\right| \leq \varepsilon .
$$

The average amplitudes of the spectrum of the functions of the horizontal $A_{a h}$ and vertical $A_{a v}$ histograms are used as informative parameters:

$$
A_{a h}=\frac{1}{K_{h}} \sum_{k=0}^{K_{h}} A_{h k} ; \quad A_{a v}=\frac{1}{K_{v}} \sum_{k=0}^{K_{v}} A_{v k} .
$$

The average amplitude of the spectrum can be used to quantitatively estimate the orientation of the surface relief elements along the axes of the image. An increase in its value is due to the greatest damage along the axis analyzed. Information on the dominant orientation of the surface defects is obtained from a comparison of the values of $A_{a h}$ and $A_{a v}$.

The presence of generalized characteristics - the average gradients $G_{h}$ and $G_{v}$ and the average amplitudes of the spectrum $A_{a h}$ and $A_{a v}$ - allows obtaining the complex integral characteristic of the image in two mutually perpendicular coordinate directions [2].

\section{FATIGUE DAMAGE ACCUMULATION IN THE FATIGUE SENSOR}

The process of fatigue damage accumulation can be divided into two main stages [17]: the stage of rapid formation of damage and the stage of saturation, which mainly involves the growth of defects that have already formed (see Fig. 1). The relative damage area can be used to obtain a quantitative estimate of the strain elements on the surface of the sensor. It reaches the highest values in the final step of the 
Table 2. Comparison of surface damage evaluation parameters

\begin{tabular}{c|c|c|c|c}
\hline \multirow{2}{*}{$\begin{array}{c}\text { Methods } \\
\text { for evaluating }\end{array}$} & \multicolumn{4}{|c}{ Damage evaluation parameters } \\
\cline { 2 - 5 } the state of the surface & $\begin{array}{c}\text { area } \\
\text { of defects }\end{array}$ & $\begin{array}{c}\text { fractal } \\
\text { dimension }\end{array}$ & $\begin{array}{c}\text { gradient spectrum } \\
\text { damage }\end{array}$ & $\begin{array}{c}\text { average amplitude } \\
\text { spectrum damage }\end{array}$ \\
\hline $\begin{array}{c}\text { Presence } \\
\text { and state } \\
\text { of damage }\end{array}$ & + & + & + & + \\
\hline $\begin{array}{c}\text { Damage } \\
\text { Localization }\end{array}$ & - & - & + & + \\
\hline
\end{tabular}

study (200,000 load cycles). Local stress relaxation occurs with simultaneously generation of local stresses in adjacent areas with activation of local shear areas (Table 1 ).

The damage area was used as an additional parameter of the state of the surface that integrally considers these physical regularities (see Fig. 6). However, it should be noted that the area is insensitive to the spatial distribution of elementary defects and cannot be used to evaluate the degree of their order. The approach proposed in this paper enables a better understanding of the kinetics of accumulation of structural-mechanical damage and the evolution of its morphology.

Surface damage was treated as a multilevel hierarchical system of ranked defects, whose evolution is aimed at minimizing the external influence at all scale levels and has spatial and temporal similarity properties [18]. Because of this, the transition to the macroscopic level of consideration was conducted without detailing the elementary acts of formation of defects on the basis of the geometrical characteristics of the structural damage. The initial step (up to 10000 cycles) involved the formation of localized shear bands in which most of the plastic deformation is concentrated. In this case, the deformation of adjacent areas has lower values, resulting in the accommodation of the plastic flow and continuity of the medium [6]. In the image (see Fig. 1a), the presence of slip bands is observed. One can see the predominance of one slip system, which is clearly reflected in the values of the damage estimate parameters (see Table 1).

After operation of 40000 cycles, the degree of damage of the sensor rapidly increases, which corresponds to the rapid growth of the vertical gradient (the horizontal gradient also increases, but at lower intensity). An increase in the number of operation cycles leads to an increase in the width and density of the slip bands and their merging into localized shear bands, which suggests the presence of strain relief elements oriented along the horizontal axis (in this case, $X^{\varphi}$ ). At the same time, the average amplitude of the spectrum of vertical histogram increases rapidly, which is reflected in the occurrence of periodic structures of strain relief in the image.

At long operation time, the horizontal gradient is half the vertical one, which indicates higher uniformity of the surface relief along the axis $X^{\varphi}$. At high loads, the gradient is somewhat reduced. This corresponds to the phase of saturation of the image with relief elements, in which the image becomes more structurally uniform, now due to the relief elements, rather than the background elements as during the initial step.

The identified kinetics of accumulation and interaction of localized shear bands in the surface layer of the fatigue sensor are consistent with the theoretical propositions in a number of papers [3, 11, 19], and the proposed approaches have certain advantages over existing methods (Table 2).

The stagewise nature of damage accumulation and the kinetics of strain localization depend on the shear stability of the material [9]. Exhaustion of ductility in cycle fatigue is directly related to the level of strain localization. This process is activated by an increase in the intensity of the shear strain and is responsible for the formation of dissipative substructures on the surface of the damaged material [20]. The approaches and computational algorithm considered here extend the capabilities of the automated evaluation of the processes of accumulation of surface defects [21].

\section{CONCLUSION}

This paper presented an algorithm for the integral evaluation of the state of surface under cyclic loading that consists of the operations of equalizing illumination, filtering, thresholding, rotation of the coordinate axes, and determining the quantitative parameters of the image. For a quantitative evaluation of the state

OPTOELECTRONICS, INSTRUMENTATION AND DATA PROCESSING Vol. 50 No. 42014 
surface, it is proposed to use a set of integral parameters: the total damage area, the average gradient along the coordinate axes, and the average amplitude of the spectrum of the histogram function.

Key features of the initiation and localization of deformation processes in the surface layers of the fatigue sensor and their relationship to the kinetics of damage accumulation were established. New features in the localization of plastic deformation in the fatigue sensor were found from numerical data on fatigue damage accumulation, which will allow improving the accuracy of evaluation of the state of structures.

\section{REFERENCES}

1. L. Molent and B. Aktepe, "Review of Fatigue Monitoring of Agile Military Aircraft," Fatigue Fract. Eng. Mater. Struct. 23 (9), 767-785 (2000).

2. P. Yasniy, P. Maruschak, and I. Konovalenko, "Measurement of Surface Mesostrain of Plastically Deformed Steel," Strain. 47 (Suppl. 2), 238-241, (2011).

3. M. V. Karuskevich, A. I. Radchenko, and E. E. Zasimchuk, "Single-Crystal as an Indicator of Fatigue Damage," Fatigue Fract. Eng. Mater. Struct. 15 (12), 1281-1283 (1992).

4. M. Petrasek, S. Ignatovich, M. Karuskevich, and T. Maslak, "Surface of Metal as an Indicator of Fatigue Damage," Adv. Military Technol. 8 (2), 83-91 (2013).

5. S. Ignatovich, M. Karuskevich, and T. Maslak, "Computer Aided Optical Method for Aircraft's Components Fatigue Life Estimation," in Mater. of the 17th European Conf. on Fracture "Multilevel Approach to Fracture of Materials, Components and Structures", Brno, Czech Republic, 2-5 Sept., 2008, pp. 2308-2313.

6. V. E. Panin, "Plastic Deformation and Fracture of Solids at the Mesoscale Level," Mater. Sci. Eng. A 234-236, 944-948 (1997).

7. V. S. Pleshanov, V. V. Kibitkin, and V. E. Panin, "Mesomechanics of Fatigue Fracture for Polycrystals with Macroconcentrators," Theor. Appl. Fract. Mech. 30 (1), 13-18 (1998).

8. P. V. Makarov, V. A. Romanova, "Mesoscale Plastic flow Generation and Development for Polycrystals," Theor. Appl. Fract. Mech. 33 (1), 1-7 (2000).

9. V. A. Romanova, R. R. Balokhonov, and S. Schmauder, "A Comparative Analysis of the Mesoscale Stress-Strain State in Two- and Three-Dimensional Polycrystalline Specimens," Phys. Mesomech. 13 (3-4), 178-183 (2010).

10. V. A. Romanova, R. R. Balokhonov, and O. S. Emelyanova, "On the Role of Internal Interfaces in the Development of Mesoscale Surface Roughness in Loaded Materials," Phys. Mesomech. 14 (3-4), 159-166 (2011).

11. M. V. Karuskevich, E. Yu.'Korchuk, T. P. Maslak, and V. M. Panteleev, "Structural Damage and Fracture of Fatigue Witness Samples," Aviatsinno-Kosmicheskaya Tekhnika i Tekhnologiya, 9 (56), 110-114, (2008).

12. M. V. Karuskevich and O. Korchuk, "Diagnostic Parameters of Fatigue of Single Crystals," AviatsionnoKosmicheskaya Tekhnika i Tekhnologiya, 10 (26), 79-83 (2005).

13. P. O. Maruschak, S. V. Panin, S. R. Ignatovich, et al., "Influence of Deformation Process in Material at Multiple Cracking and Fragmentation of Nanocoating," Theor. Appl. Fract. Mech. 57 (1), 43-48 (2012).

14. J.-H. Park, M. S. Myung, and Y.-J. Kim, "Tensile and High Cycle Fatigue Test of Al-3\% Ti Thin Film," Sensors and Actuators A: Phys. 147 (2), 561-569 (2008).

15. P. Yasniy, P. Maruschak, I. Konovalenko, et al., "Multiple Cracks on Continuous Caster Rolls Surface: A ThreeDimensional View," in Proc. of the 4th Intern. Conf. "Processing and Structure of Materials", Palic, Serbia, 2010, pp. $7-12$.

16. R. O. Duda and P. E. Hart, "Use of the Hough Transformation to Detect Lines and Curves in Pictures," Commun. ACM. 15 (1), 11-15 (1972).

17. S. V. Panin, A. V. Byakov, P. S. Lyubutin, et al., "Multiscale Method for Studying the Deformation and Fracture of Loaded Solids from Data on Acoustic Emission, Digital Image Correlation, and Strain Measurement," Zavod. Lab., Diagnostika Materialov 77 (9), 50-59 (2011).

18. P. V. Yasniy, P. O. Maruschak, S. V. Panin, and P. S. Lyubutin, "Mesomechanics and Hierarhical Levels of Fatigue Crack Growth in 25Cr1Mo1V Steel," Intern. J. Terraspace Sci. Eng. 3 (Is. 1), 169-175 (2011).

19. M. V. Karuskevych, I. M. Zhuravel', and T. P. Maslak, "Application of Fractal Geometry to the Problems of Prediction of the Residual Service Life of Aircraft Structures," Mater. Sci. 47 (5), 621-626 (2012).

20. S. Siriwardane, M. Ohga, R. Dissanayake, and K. Taniwaki, "Application of New Damage Indicator-Based Sequential Law for Remaining Fatigue Life Estimation of Railway Bridges," J. Constructional Steel Research. 64 (2), 228-237 (2008).

21. I. V. Konovalenko and P. O. Marushchak, "Error Analysis of an Algorithm for Identifying Thermal Fatigue Cracks," Avtometriya 47 (4), 49-57 (2011) [Optoelectron., Instrum. Data Process. 47 (4), 360-367 (2011)].

OPTOELECTRONICS, INSTRUMENTATION AND DATA PROCESSING Vol. $50 \quad$ No. 42014 